\title{
THE COSMIC-RAY ENERGY SPECTRUM OBSERVED WITH THE SURFACE DETECTOR OF THE TELESCOPE ARRAY EXPERIMENT
}

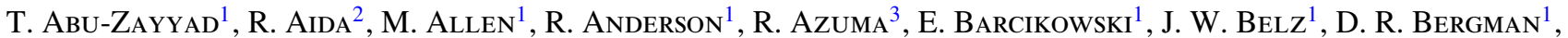

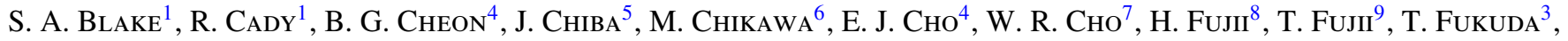

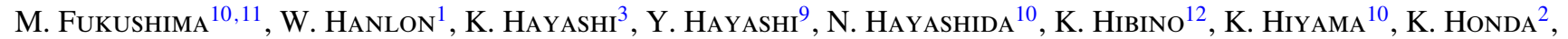

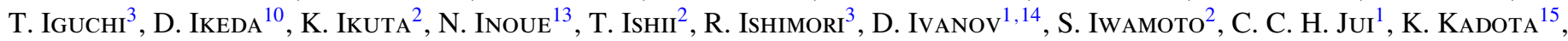

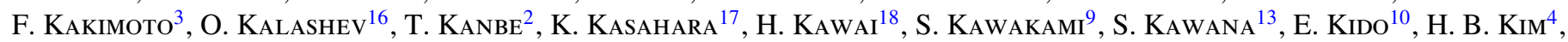
H. K. Kim ${ }^{7}$, J. H. Kim ${ }^{1}$, J. H. Kim ${ }^{4}$, K. Kitamoto ${ }^{6}$, S. Kitamura ${ }^{3}$, Y. Kitamura ${ }^{3}$, K. Kobayashi ${ }^{5}$, Y. Kobayashi ${ }^{3}$, Y. Kondo ${ }^{10}$,

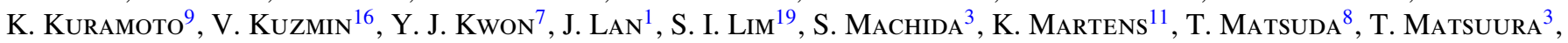
T. Matsuyama ${ }^{9}$, J. N. Matthews ${ }^{1}$, M. Minamino ${ }^{9}$, K. Miyata ${ }^{5}$, Y. Murano ${ }^{3}$, I. Myers ${ }^{1}$, K. Nagasawa ${ }^{13}$, S. Nagataki ${ }^{20}$,

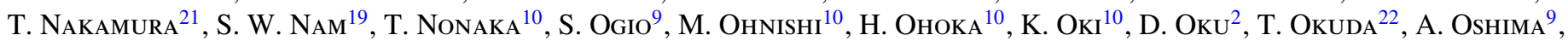
S. Ozawa ${ }^{17}$, I. H. Park ${ }^{19}$, M. S. Pshirkov ${ }^{23}$, D. C. RodrigueZ ${ }^{1}$, S. Y. RoH $^{24}$, G. RubTsov ${ }^{16}$, D. Ryu ${ }^{24}$, H. SaGawa ${ }^{10}$, N. Sakurai ${ }^{9}$, A. L. Sampson ${ }^{1}$, L. M. Scott ${ }^{14}$, P. D. Shah ${ }^{1}$, F. Shibata ${ }^{2}$, T. Shibata ${ }^{10}$, H. Shimodaira ${ }^{10}$, B. K. Shin ${ }^{4}$, J. I. Shin ${ }^{7}$, T. Shirahama ${ }^{13}$, J. D. Smith ${ }^{1}$, P. Sokolsky ${ }^{1}$, B. T. Stokes ${ }^{1}$, S. R. Stratton ${ }^{1}, 14$, T. Stroman ${ }^{1}$, S. SuZUKi ${ }^{8}$,

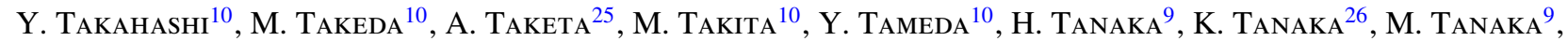

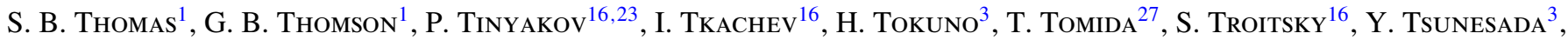

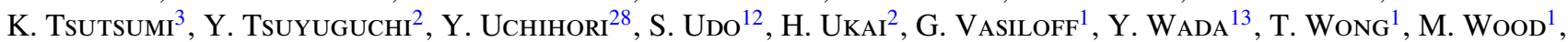

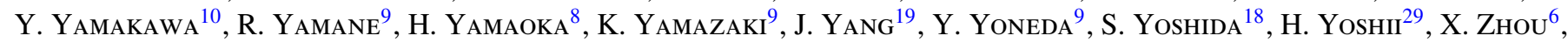
R. ZOLLINGER ${ }^{1}$, AND Z. ZUNDEL ${ }^{1}$

${ }^{1}$ High Energy Astrophysics Institute and Department of Physics and Astronomy, University of Utah, Salt Lake City, Utah, USA

${ }^{2}$ University of Yamanashi, Interdisciplinary Graduate School of Medicine and Engineering, Kofu, Yamanashi, Japan

${ }^{3}$ Graduate School of Science and Engineering, Tokyo Institute of Technology, Meguro, Tokyo, Japan

${ }^{4}$ Department of Physics and The Research Institute of Natural Science, Hanyang University, Seongdong-gu, Seoul, Republic of Korea

${ }^{5}$ Department of Physics, Tokyo University of Science, Noda, Chiba, Japan

${ }^{6}$ Department of Physics, Kinki University, Higashi Osaka, Osaka, Japan

${ }^{7}$ Department of Physics, Yonsei University, Seodaemun-gu, Seoul, Republic of Korea

${ }^{8}$ Institute of Particle and Nuclear Studies, KEK, Tsukuba, Ibaraki, Japan

${ }^{9}$ Graduate School of Science, Osaka City University, Osaka, Osaka, Japan

${ }^{10}$ Institute for Cosmic Ray Research, University of Tokyo, Kashiwa, Chiba, Japan

${ }^{11}$ Kavli Institute for the Physics and Mathematics of the Universe, University of Tokyo, Kashiwa, Chiba, Japan

${ }^{12}$ Faculty of Engineering, Kanagawa University, Yokohama, Kanagawa, Japan

${ }^{13}$ The Graduate School of Science and Engineering, Saitama University, Saitama, Saitama, Japan

${ }^{14}$ Department of Physics and Astronomy, Rutgers University, Piscataway, USA

${ }^{15}$ Department of Physics, Tokyo City University, Setagaya-ku, Tokyo, Japan

${ }^{16}$ Institute for Nuclear Research of the Russian Academy of Sciences, Moscow, Russia

${ }^{17}$ Advanced Research Institute for Science and Engineering, Waseda University, Shinjuku-ku, Tokyo, Japan

${ }^{18}$ Department of Physics, Chiba University, Chiba, Chiba, Japan

${ }^{19}$ Department of Physics and Institute for the Early Universe, Ewha Womans University, Seodaaemun-gu, Seoul, Republic of Korea

${ }^{20}$ Yukawa Institute for Theoretical Physics, Kyoto University, Sakyo, Kyoto, Japan

${ }^{21}$ Faculty of Science, Kochi University, Kochi, Kochi, Japan

${ }^{22}$ Department of Physical Sciences, Ritsumeikan University, Kusatsu, Shiga, Japan

${ }^{23}$ Service de Physique Théorique, Université Libre de Bruxelles, Brussels, Belgium

${ }^{24}$ Department of Astronomy and Space Science, Chungnam National University, Yuseong-gu, Daejeon, Republic of Korea

${ }^{25}$ Earthquake Research Institute, University of Tokyo, Bunkyo-ku, Tokyo, Japan

${ }^{26}$ Department of Physics, Hiroshima City University, Hiroshima, Hiroshima, Japan

${ }^{27}$ RIKEN, Advanced Science Institute, Wako, Saitama, Japan

${ }^{28}$ National Institute of Radiological Science, Chiba, Chiba, Japan

${ }^{29}$ Department of Physics, Ehime University, Matsuyama, Ehime, Japan

Received 2013 February 28; accepted 2013 March 25; published 2013 April 10

\section{ABSTRACT}

The Telescope Array (TA) collaboration has measured the energy spectrum of ultra-high energy cosmic rays (UHECRs) with primary energies above $1.6 \times 10^{18} \mathrm{eV}$. This measurement is based upon four years of observation by the surface detector component of TA. The spectrum shows a dip at an energy of $4.6 \times 10^{18} \mathrm{eV}$ and a steepening at $5.4 \times 10^{19} \mathrm{eV}$ which is consistent with the expectation from the GZK cutoff. We present the results of a technique, new to the analysis of UHECR surface detector data, that involves generating a complete simulation of UHECRs striking the TA surface detector. The procedure starts with shower simulations using the CORSIKA Monte Carlo program where we have solved the problems caused by use of the "thinning" approximation. This simulation method allows us to make an accurate calculation of the acceptance of the detector for the energies concerned.

Key words: astroparticle physics - cosmic rays - methods: data analysis

Online-only material: color figures 


\section{INTRODUCTION}

One of the most powerful tools for studying the origin of ultrahigh energy cosmic rays (UHECRs) is their energy spectrum, which manifests several features that reveal important information about the cosmic rays, their sources, and their propagation across cosmological distances. One example is the high-energy $\left(4-6 \times 10^{19} \mathrm{eV}\right)$ suppression in the spectrum which was predicted by Greisen (1966) and by Zatsepin \& Kuzmin (1966), and is called the GZK cutoff. These authors predicted a strong suppression in the spectrum due to the interaction of cosmic rays with photons of the cosmic microwave background radiation. They pointed out that a spectrum suppression is expected in both cosmic protons (by photo-pion production) and heavier nuclei (by spallation) for particles traveling more than 50 $\mathrm{Mpc}$ from their sources. If cosmic rays are protons there should also be a dip in the spectrum, caused by $e^{+} e^{-}$pair production in the same interactions, at an energy of about $5 \times 10^{18} \mathrm{eV}$ (Berezinsky \& Grigor'eva 1988). For heavier nuclei, interactions with the background photon flux do not cause such a dip.

The AGASA experiment (Takeda et al. 1998, 2003), comprised of a surface array of 111 scintillation counters, was the first detector to be large enough to test this theory with sufficient statistics. However, they did not observe the suppression. The first experiment to observe the GZK cutoff was the High Resolution Fly's Eye (HiRes) experiment (Abbasi et al. 2008), which consisted of fluorescence detectors located atop two desert mountains in western Utah. HiRes reported a cutoff energy of $(5.6 \pm 0.5 \pm 0.9) \times 10^{19} \mathrm{eV}$, which is consistent with a suppression of protons. They also observed the ankle structure: a hardening of the spectrum at an energy of $4.5 \pm 0.05 \pm 0.8 \times 10^{18} \mathrm{eV}$ as expected for cosmic protons. (For both of these values, the first uncertainty is statistical and the second systematic.) HiRes also published measurements of the shower maximum slant depth $\left(X_{\max }\right)$ that indicated a predominately light composition above $2 \times 10^{18} \mathrm{eV}$ (Abbasi et al. 2010).

A somewhat different picture is seen by the Pierre Auger Observatory (PAO), located in Argentina. The PAO consists of a surface detector (SD) of 1600 water tanks, accompanied by 24 fluorescence telescopes which are equally apportioned between four sites located at the SD corners. The PAO also observes the high-energy suppression, but at $(2.9 \pm 0.2) \times 10^{19} \mathrm{eV}$ (Abraham et al. 2008, 2010b). They also see the ankle, but their $X_{\max }$ results may indicate that the composition is heavy at the highest energies (Abraham et al. 2010a). One possible interpretation of the PAO results is that the high-energy suppression is caused by spallation of heavy nuclei. The cause of the ankle would need to be explained by a separate mechanism.

The Telescope Array (TA) experiment, also in western Utah, is the largest experiment studying UHECRs in the northern hemisphere. A layout of the TA experiment is shown in Figure 1. It consists of a surface detector of 507 scintillation counters (Abu-Zayyad et al. 2012b), plus 38 fluorescence telescopes (Tokuno et al. 2012; Abu-Zayyad et al. 2012a) located at three sites overlooking the SD. Twenty-four of the telescopes, deployed at the two southern sites, were newly built for the experiment, and the fourteen telescopes at the northern site are reconditioned HiRes telescopes. TA combines the experimental techniques of AGASA and HiRes, in order to understand the difference between their results.

This Letter reports on a measurement of the cosmic-ray spectrum above $1.6 \times 10^{18} \mathrm{eV}$ made by the TA SD over

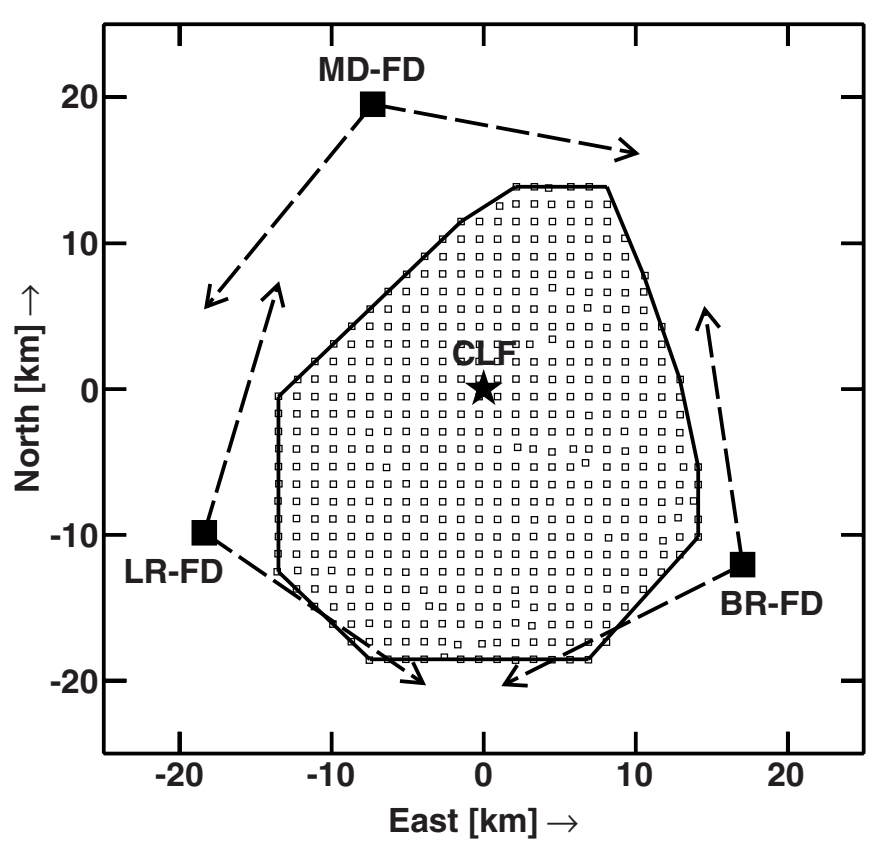

Figure 1. Layout of the Telescope Array experiment. This figure shows the 507 surface detector counters deployed on a $1.2 \mathrm{~km}$ grid. Fluorescence telescopes overlook the surface detector at three sites called Middle Drum (MD) on the north, Long Ridge (LR) on the southwest, and Black Rock (BR) on the southeast. The Central Laser Facility (CLF) is situated in the center of array, equidistant from all three fluorescence detectors.

approximately four years of observation between 2008 May 11 and 2012 May 20. For this study, we used an analysis method that, while standard for fluorescence detectors, is being successfully implemented for the first time for a surface array studying cosmic rays in the ultra-high $(>1 \mathrm{EeV})$ energy regime. Instead of restricting our analysis to a domain where we expect $100 \%$ efficiency, as has been done by previous surface detector experiments at these energies, the TA SD detector aperture is calculated using extensive air showers generated in detail by the CORSIKA simulation package (Heck et al. 1998), accompanied by a full GEANT simulation of the detector (Allison et al. 2006). Another important aspect of this technique, new to surface detectors operating in the ultra-high energy regime, is the validation of the simulation by comparisons of key distributions from the data to those obtained from the Monte Carlo (MC) simulation. Moreover, our study overcomes the inability of "thinned" simulated showers (e.g., as used in CORSIKA and AIRES; Sciutto 2002) to reproduce the particle density and arrival time fluctuations far from the core. The solution applied is a novel dethinning technique that replicates a non-thinned simulation (Stokes et al. 2012) at the lateral core distances where most of the detector data is collected.

\section{THE TA SURFACE DETECTOR}

Each counter of the TA SD consists of two layers of $1.2 \mathrm{~cm}$ thick plastic scintillator, both $3 \mathrm{~m}^{2}$ in area. Photons produced by ionizing particles passing through the counters are collected by wavelength shifting fibers and read out by photomultiplier tubes, one for each layer. A histogram of pulse heights, triggered by a coincidence between the two layers within an individual $\mathrm{SD}$, is collected every 10 minutes. This histogram is dominated by single muons with a count rate of $\sim 700 \mathrm{~Hz}$. Each 10 minute histogram is used to calibrate the associated scintillator to the pulse height of a minimum ionizing particle (MIP) with $\sim 1 \%$ 


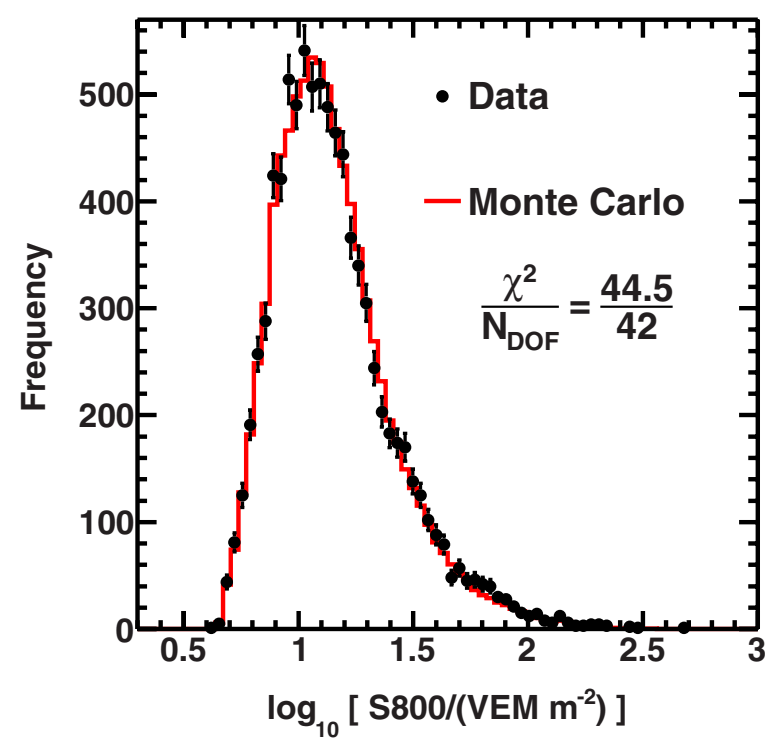

Figure 2. Data and MC comparison of the event $S(800)$ distributions. The reduced $\chi^{2}$ is 1.06 , indicating a good agreement.

(A color version of this figure is available in the online journal.)

accuracy. The SD array trigger requires at least three adjacent counters with pulse heights over 3 MIP to fire within $8 \mu \mathrm{sec}$. A $50 \mathrm{MHz}$ flash analog-to-digital converter readout system then saves the signal traces for all counters in the array with more than 0.3 MIP. Two fits are used to reconstruct the properties of the cosmic ray. First, a fit to the times that counters were struck, using the modified Linsley shower-shape function (Teshima et al. 1986), is made to determine the arrival direction and the core position of the event. Subsequently, a lateral distribution fit, with the same functional form used by the AGASA experiment (Takeda et al. 1998, 2003), is employed to find $S(800)$, the density of shower particles at a lateral distance of $800 \mathrm{~m}$ from the core. The energy is then estimated by using a look-up table in $S(800)$ and zenith angle determined from an exhaustive MC simulation.

\section{APERTURE CALCULATION}

In the ultra-high energy regime, computer-time requirements make it impossible to follow every particle when simulating showers. An approximation called thinning is used in programs like CORSIKA and AIRES to reduce the computational load by only performing a small, statistically representative sample of the air shower simulation. Thinned showers can be used for simulation of fluorescence detectors because the fluorescence light comes mostly from near the shower axis where the particle density is extremely high, and the fluctuations in the signal are dominated by the Poisson nature of fluorescence photon statistics. But for surface detectors, which operate far from the shower core, the number of shower particles is low and the thinning approximation fails to represent the intrinsic density fluctuations within the shower. To simulate the TA SD accurately, we have developed a procedure called "dethinning," where we statistically regenerate each group of thinned particles from its weighted representative (Stokes et al. 2012).

The MC simulation of the TA SD has the goal of making an accurate representation of the data and our detectors. We start with a library of showers generated by the CORSIKA program using QGSJET-II-03 (Ostapchenko 2006) to model high-energy hadronic interactions, FLUKA (Ferrari et al. 2005;

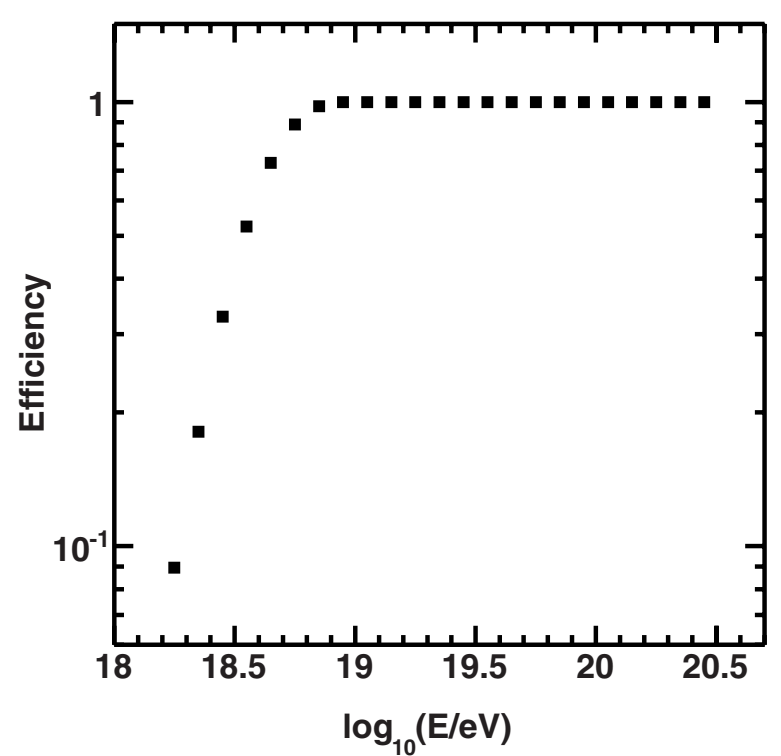

Figure 3. Efficiency as a function of energy. Both trigger and reconstruction effects are included.

Battistoni et al. 2007) to model low-energy hadronic interactions, and EGS4 (Nelson et al. 1985) to model electromagnetic interactions. For this library, proton showers were used exclusively because both the HiRes composition results (Abbasi et al. 2010) and the preliminary TA composition result (Tameda et al. 2011) are consistent with protons generated by QGSJET-II-03. A complete representation of calibration and ontime for each surface counter as a function of time is also included. Events are then chosen from our shower libraries according to the spectrum previously measured by the HiRes collaboration (Abbasi et al. 2008). Direct comparisons between data and MC show that the result closely resembles the data (T. Abu-Zayyad et al., in preparation). Figure 2 shows a comparison of the $S(800)$ of cosmic-ray showers. The excellent agreement between the data and simulation exemplifies the accuracy of our simulation and the resulting efficiency calculation of the SD.

The selection criteria employed in our analysis are as follows:

1. Each event must include at least five counters.

2. The reconstructed primary zenith angle must be less than $45^{\circ}$.

3. The reconstructed event core must be more than $1200 \mathrm{~m}$ from edge of the array.

4. Both the timing and lateral distribution fits must have $\chi^{2} /$ degree of freedom value less than 4 .

5. The angular uncertainty estimated by the timing fit must be less than $5^{\circ}$.

6. The fractional uncertainty in $S(800)$ estimated by the lateral distribution fit must be less than $25 \%$.

Between 2008 May 8 and 2012 May 20, 13,100 events above $10^{18.2} \mathrm{eV}$ were collected that satisfy these criteria. Figure 3 shows the efficiency of reconstruction calculated from the TA SD Monte Carlo Program. The values of aperture and exposure for this data set, corresponding to the $100 \%$ efficiency region, are $920 \mathrm{~km}^{2} \mathrm{sr}$ and $3690 \mathrm{~km}^{2} \mathrm{sr} \mathrm{yr}$, respectively. For energies above $10^{18.2} \mathrm{eV}$ (where the efficiency is $\sim 10 \%$ of its plateau value) we can accurately simulate all air showers, both welland poorly-reconstructed. The resolution of the TA SD energy determination is better than $20 \%$ above $10^{19} \mathrm{eV}$.

The uncertainty in energy scale of the MC simulation of an $\mathrm{SD}$ is large, and possible biases associated with the modeling 


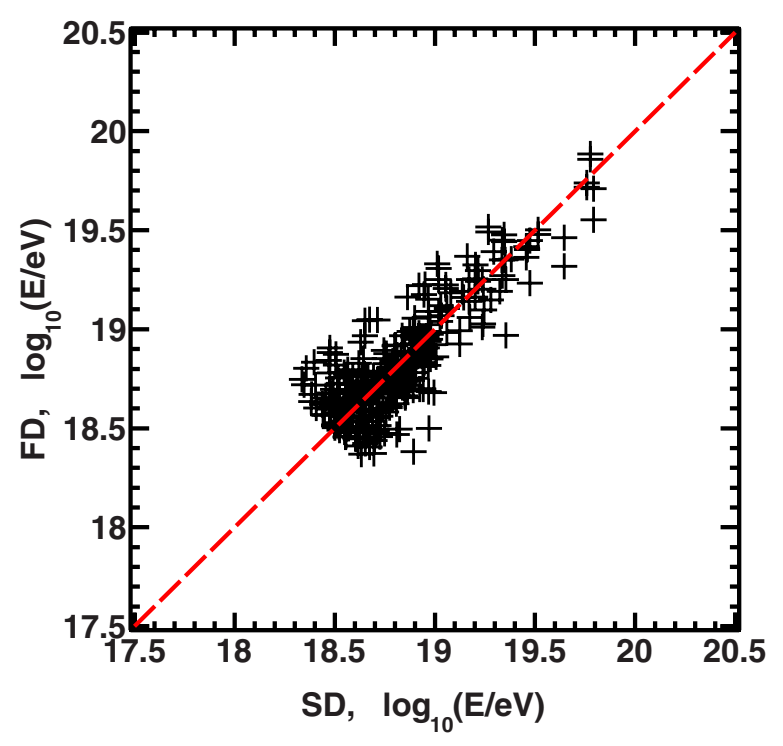

Figure 4. Energy comparison between the TA SD and FD after the 27\% normalization has been applied to the SD.

(A color version of this figure is available in the online journal.)

of hadronic interactions (e.g., from extrapolations of cross sections measured at much lower energies) are difficult to determine. However, the energy scale uncertainty is experimentally well-controlled for a fluorescence detector (FD) since the energy measurement is calorimetric. We therefore correct our energy scale to the TA FD using events seen in common between the FD and SD. The observed differences between the FD and SD events are well described by a simple proportionality relationship, where the SD energy scale is $27 \%$ higher than the FD. Figure 4 shows a scatter plot of FD versus SD energies, where the latter have been rescaled. Events from all three FD stations were included in this plot. The two southern FD stations were calibrated using independent techniques from the northern station, which consists of reconditioned HiRes fluorescence telescopes. The resulting energy scales are consistent for all TA fluorescence detectors.

\section{SPECTRUM}

Figure 5 shows the spectrum measured by the TA SD, where the differential flux, $J(E)=d^{4} N(E) / d E d A d \Omega d t$ is multiplied by $E^{3}$, and plotted against $\log _{10} E$. The ankle structure and the suppression at the highest energies are clearly visible. A fit to a broken power law (BPL) determines the energies of these features. The fit finds the ankle at an energy of $(4.6 \pm 0.3) \times 10^{18} \mathrm{eV}$ and the suppression at $(5.4 \pm 0.6) \times$ $10^{19} \mathrm{eV}$. The power exponents for the three regions (below the ankle, between the breaks, and above the suppression) are $-3.34 \pm 0.04,-2.67 \pm 0.03$, and $-4.6 \pm 0.6$ respectively. Also shown in Figure 5 are the spectra reported by AGASA (Takeda et al. 2003), HiRes (monocular mode; Abbasi et al. 2008), and PAO (combined hybrid and SD; Abraham et al. 2010b). The HiRes and TA SD spectra agree very well, both in the energy region above $10^{18.85} \mathrm{eV}$ where the TA SD is $100 \%$ efficient, and also at lower energies where TA employs a substantial efficiency correction.

A linear extrapolation of the power law below the suppression predicts 58.6 events above the break; whereas TA observed only 21 events. This difference corresponds to a Poisson probability of $1.44 \times 10^{-8}$, or 5.5 standard deviations significance. A

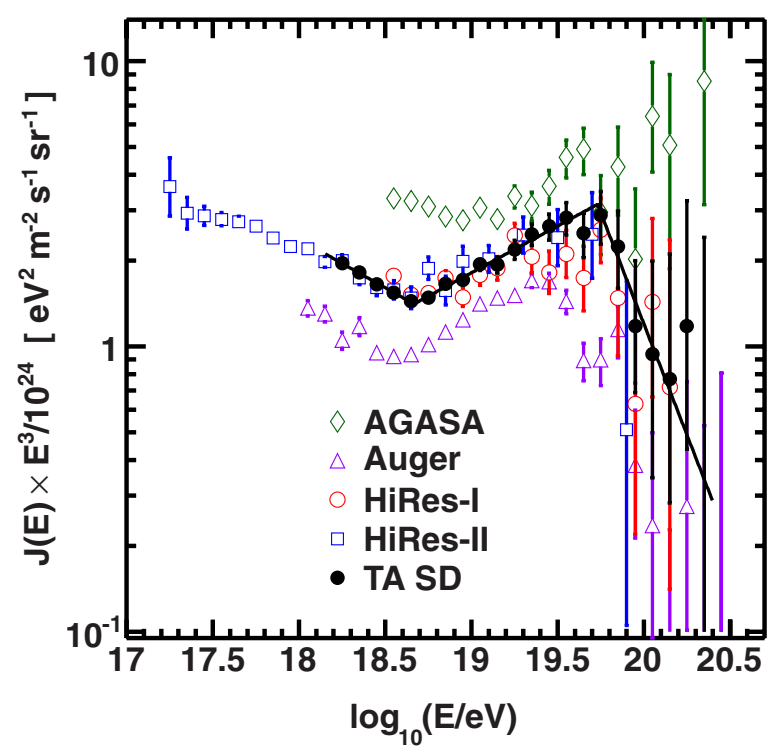

Figure 5. Cosmic-ray flux multiplied by $\mathrm{E}^{3}$. The solid line shows the fit of the TA SD data to a broken power law.

(A color version of this figure is available in the online journal.)

related observable, $E_{1 / 2}$, is the energy at which the integral spectrum falls to one-half of its expected value in the absence of the GZK cutoff. Under a wide range of assumptions about the spectrum of extragalactic sources, $E_{1 / 2}$ is predicted to be $10^{19.72} \mathrm{eV}$ for protons (Berezinsky et al. 2006). HiRes reported $\log _{10} E=19.73 \pm 0.07$ (Abbasi et al. 2008). We measure $\log _{10} E=19.72 \pm 0.05$.

This 5.5 standard deviation observation provides independent confirmation of the GZK cutoff observed by HiRes (Abbasi et al. 2008). Furthermore, the energy of the cutoff is consistent with the interpretation that the composition is protonic.

T. Abu-Zayyad et al. (in preparation) includes a description of systematic uncertainties in the SD spectrum measurement. The largest source of systematic uncertainty in the spectrum is that of the energy scale. Since the SD energy scale is fixed to that of the TA fluorescence detectors, we take the systematic uncertainty in the SD energy to be 22\% (Abu-Zayyad et al. 2011), the same as the FD. This propagates into a $37 \%$ uncertainty in the flux. We estimate the systematic uncertainty in the aperture calculation by removing the event selection criteria, one by one, and measuring the ratio of the number of events in the data and in the MC simulation. This ratio does not change by more than $3 \%$ in any energy bin above $10^{18.2} \mathrm{eV}$, so we assign this value to be the systematic uncertainty in the aperture.

\section{CONCLUSIONS}

We have measured the spectrum of cosmic rays in the energy range $10^{18.2}-10^{20.3} \mathrm{eV}$ using the surface detector of the TA experiment. In the analysis, we have introduced a technique, new to the ultra-high energy regime for surface detectors, of calculating the surface detector aperture using MC simulation, which allows us to measure the spectrum even when the SD efficiency is less than $100 \%$. This technique includes a dethinning process that enables the simulation of air showers with excellent detail. We found that the energy scale of the SD determined from simulations can be reconciled with the calorimetric scale of fluorescence detectors by a simple renormalization of $27 \%$. 
Two features are seen in the spectrum, the ankle and the high-energy suppression. Fitting the spectrum to a broken power law shows a definite break at an energy of $(5.4 \pm 0.6) \times 10^{19} \mathrm{eV}$, which is consistent with the GZK cutoff energy expected for protons. An extended spectrum beyond the GZK energy is ruled out with a statistical significance of 5.5 standard deviations. Our result is in excellent agreement with that of the HiRes experiment where fluorescence detectors were used. This result demonstrates, contrary to the AGASA claim, that there is no difference between measurements of the cosmic-ray spectrum using a fluorescence detector and a surface scintillation array once the energy scales are normalized.

In summary, by combining the two techniques of surface detectors and fluorescence detectors (used by the AGASA and HiRes experiments), we have now obtained a consistent energy spectrum for UHECRs from both techniques. The spectrum obtained by our experiment demonstrates spectral features, a dip and a cutoff, consistent with the interaction of extra-galactic protons with the cosmic microwave background (GZK process). Finally, if we account for a $20 \%$ systematic difference in energy scale, our measurement is in good agreement with the spectrum reported by PAO with one exception: the GZK break is reported at $(2.9 \pm 0.2) \times 10^{19} \mathrm{eV}$ by PAO (Abraham et al. 2010b); even with a $20 \%$ energy scale correction the difference between the TA and PAO measurements is three standard deviations.

The Telescope Array experiment is supported by the Japan Society for the Promotion of Science through Grants-inAids for Scientific Research on Specially Promoted Research (21000002) "Extreme Phenomena in the Universe Explored by Highest Energy Cosmic Rays" and for Scientific Research (S) (19104006), and the Inter-University Research Program of the Institute for Cosmic Ray Research; by the U.S. National Science Foundation awards PHY-0307098, PHY-0601915, PHY-0703893, PHY-0758342, and PHY-0848320 (Utah), and PHY-0649681 (Rutgers); by the National Research Foundation of Korea (2006-0050031, 2007-0056005, 2007-0093860, 2010-0011378, 2010-0028071, R32-10130, 2011-0002617); by the Russian Academy of Sciences, RFBR grants 10-02-01406a and 11-02-01528a (INR), IISN project No. 4.4509.10 and Belgian Science Policy under IUAP VI/11 (ULB). The foundations of Dr. Ezekiel R. and Edna Wattis Dumke, Willard L. Eccles and the George S. and Dolores Dore Eccles all helped with generous donations. The State of Utah supported the project through its Economic Development Board, and the University of Utah through the Office of the Vice President for Research. The experimental site became available through the cooperation of the Utah School and Institutional Trust Lands Administration (SITLA), U.S. Bureau of Land Management, and the U.S. Air Force. We also thank the people and the officials of Millard County, Utah for their steadfast and warm support. We gratefully acknowledge the contributions from the technical staffs of our home institutions. An allocation of computer time from the Center for High Performance Computing at the University of Utah is gratefully acknowledged.

\section{REFERENCES}

Abbasi, R. U., Abu-Zayyad, T., Allen, M., et al. 2008, PhRvL, 100, 101101 Abbasi, R. U., Abu-Zayyad, T., Al-Seady, M., et al. 2010, PhRvL, 104,161101

Abraham, J., Abreu, P., Aglietta, M., et al. 2008, PhRvL, 101, 061101

Abraham, J., Abreu, P., Aglietta, M., et al. 2010a, PhRvL, 104, 091101

Abraham, J., Abreu, P., Aglietta, M., et al. 2010b, PhLB, 685, 239

Abu-Zayyad, T., Aida, R., Allen, M., et al. 2012a, APh, 39, 109

Abu-Zayyad, T., Aida, R., Allen, M., et al. 2012b, NIMPA, 689, 87

Abu-Zayyad, T., Allen, M., Barcikowski, E., et al. 2011, in Proceedings of the $32^{\text {nd }}$ ICRC, Vol. 2, Beijing, 250

Allison, J., Amako, K., Apostolakis, J., et al. 2006, ITNS, 53, 270

Battistoni, G., Cerutti, F., Fassò, A., et al. 2007, in AIP Conf. Proc. 896, Hadronic Shower Simulation Workshop (Melville, NY: AIP), 31

Berezinsky, V., Gazizov, A., \& Grigorieva, S. 2006, PhRvD, 74, 043005

Berezinsky, V. S., \& Grigor' eva, S. I. 1988, A\&A, 199, 1

Ferrari, A., Sala, P. R., Fasso, A., \& Ranft, J. 2005, FLUKA: A Multi-Particle Transport Code (Program version 2005), Tech. Rep. 2005-010, CERN

Greisen, K. 1966, PhRvL, 16, 748

Heck, D., Schatz, G., Thouw, T., Knapp, J., \& Capdevielle, J. N. 1998, CORSIKA: A Monte Carlo Code to Simulate Extensive Air Showers, Tech. Rep. 6019, FZKA

Nelson, W. R., Hirayama, H., \& Rogers, D. W. O. 1985, The EGS4 Code System, Tech. Rep. 0265, SLAC

Ostapchenko, S. 2006, NuPhS, 151, 143

Sciutto, S. J. 2002, AIRES: A System for Air Shower Simulations, Tech. Rep. 2.6.0, UNLP

Stokes, B. T., Cady, R., Ivanov, D., Matthews, J. N., \& Thomson, G. B. 2012, $\mathrm{APh}, 35,759$

Takeda, M., Hayashida, N., Honda, K., et al. 1998, PhRvL, 81, 1163

Takeda, M., Sakaki, N., Honda, K., et al. 2003, APh, 19, 447

Tameda, Y., Fukushima, M., Ikeda, D., et al. 2011, in Proceedings of the $32^{\text {nd }}$ ICRC, Vol. 2, Beijing, 246

Teshima, M., Matsubara, Y., Hara, T., et al. 1986, JPhG, 12, 1097

Tokuno, H., Tameda, Y., Takeda, M., et al. 2012, NIMPA, 676, 54

Zatsepin, G. T., \& Kuzmin, V. A. 1966, JETPL, 4, 78 